\title{
AN EXTENSION OF THE WEYL-VON NEUMANN THEOREM TO NORMAL OPERATORS $\left({ }^{1}\right)$
}

\author{
BY \\ I. DAVID BERG
}

\begin{abstract}
We prove that a normal operator on a separable Hilbert space can be written as a diagonal operator plus a compact operator. If, in addition, the spectrum lies in a rectifiable curve we show that the compact operator can be made HilbertSchmidt.
\end{abstract}

In 1909 Hermann Weyl proved [3] that each bounded Hermitian operator on a separable Hilbert space can be written as the sum of a diagonal operator and a compact operator. J. von Neumann proved in 1935 [2] that the compact operator can actually be made Hilbert-Schmidt and also observed that boundedness is unnecessary. P. R. Halmos has inquired in [1] whether the Weyl result, and, in his 1969 Brazilian lectures, whether the von Neumann result can be extended to normal operators.

We prove here that Weyl's result can be so extended. That is, each normal operator can be written as the sum of a diagonal operator and a compact operator. If, in addition, the spectrum lies in a rectifiable curve, von Neumann's result holds. That is, the compact operator can actually be made Hilbert-Schmidt. In each of these cases the bounded case is the crucial one; the extension to unbounded operators is easy. It is also easy to see that, as in the Weyl and von Neumann results, the norm of the compact operator or the Hilbert-Schmidt norm of the Hilbert-Schmidt operator respectively may be made arbitrarily small.

We conjecture that, in general, for normal operators, the compact operator cannot be made Hilbert-Schmidt. Indeed, we conjecture that the operator given by multiplication by $z$ on $L^{2}[I \times I]$ cannot be so decomposed; the positive 2 dimensional Lebesgue measure of the purely continuous spectrum of this operator seems to prevent the compact operator from being Hilbert-Schmidt.

We were first made acquainted with these questions by a lecture of P. R. Halmos at the regional conference sponsored by the NSF at Texas Christian University in May 1970. We gratefully acknowledge valuable discussion with J. Dyer, P. Halmos and H. Porta.

We use standard terminology. However, for economy of notation we will allow ourselves the looseness of speaking of an unbounded operator "on" $H$. We use

Received by the editors October 8, 1970.

AMS 1970 subject classifications. Primary 47B15, 47B05, 47B10.

Key words and phrases. Normal operator, diagonal operator, Hilbert-Schmidt, compact.

(1) This research was partially supported by NSF grant NSF GP-14785.

Copyright (C) 1971, American Mathematical Society 
the spectral theorem for normal operators, $A=\int_{\sigma(A)} z d E_{z}$, essentially as our definition. The definition of diagonal may be unfamiliar. We say that an operator $A$ on a separable Hilbert space is diagonal if there exists a complete orthonormal set of eigenvectors for $A$.

We will prove Theorem 1 in considerable detail. The remaining theorems just modify and exploit the technique of Theorem 1; therefore in these we will merely indicate the nontrivial modifications.

Our proof has features in common with both the Weyl and the von Neumann proof. The idea of constructing the eigenvectors by using small pieces of the spectrum is natural and common to all our proofs. The von Neumann proof is pellucid and thoroughly modern. We do not see, even after modifying the obviously unextendable Hilbert-Schmidt arguments, how to extend his proof to the normal case. In one elegant step ( $\$ 2$, p. 9 of [2]) he exploits the selfadjointness in a way that has no analogue for normal operators. As Halmos points out, his own proof [1] encounters a similar difficulty. The Weyl proof is much less clear. While he, too, has several steps which require selfadjointness it is not obvious that they could not be wrenched into a suitable form to serve in the normal case.

Our proof of Theorem 1 can be slightly simplified as was kindly pointed out by B. E. Johnson. We sketch this simplification after our proof. However, this simplification does not allow the convenient extension to the Hilbert-Schmidt remainder which we achieve in Theorem 3 .

THEOREM 1. Let A be a bounded normal operator on the separable Hilbert space $H$. Then $A=D+C$ where $D$ is diagonal and $C$ is compact.

Proof. We write $A=\int_{\sigma(A)} z d E_{z}$. We may assume $\sigma(A)$ lies in the unit square. We form a sequence of partitions of $\sigma(A)$ by partitioning the square as follows:
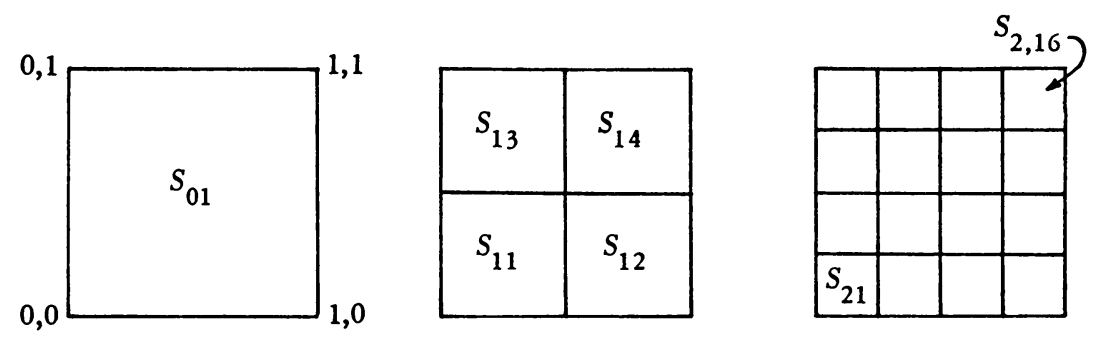

We take the $S_{i j}$ to be open on the right and top, closed on the left and bottom except where the right and/or top abut the boundary of the unit square. We denote by $P_{i j}$ the value of the spectral measure $d E_{z}$ on the Borel set $S_{i j}$. We will denote $P_{i j}(H)$ as $H_{i j}$. It is perfectly possible for some $H_{i j}$ to be 0 .

We may now suppose that $\omega_{1}, \omega_{2}, \ldots$ is an orthonormal basis for $H$.

Let us try to describe a major step in our construction and proof before we actually write out the fully indexed construction. 
We let $\Psi^{(1)}=\omega_{1}$. We will let $H_{1}=\bigvee_{i, j} P_{i j} \Psi^{(1)}$. That is, $H_{1}$ is the space spanned by all projections in subsquares of $\Psi^{(1)}$. We now form a special sort of orthonormal basis $\left\{\varphi_{n}\right\}$ for $H_{1}$ consisting of vectors with support in smaller and smaller subsquares. The essential point in this construction is the observation that once we have spanned the projection in a subsquare $Q$ of side $2^{-n}$, then in order to obtain the projections on the 4 subsquares of side $2^{-(n+1)}$ we need add only 3 vectors and these vectors can be chosen with support in $Q$.

This then gives us, for a covering of the square by the $2^{2 n}$ subsquares of side $2^{-n}$, a set of $3 \cdot 2^{2 n}$ vectors in $\left\{\varphi_{n}\right\}$ with at most 3 vectors in each such subsquare. These vectors, when adjoined to the set of $2^{2 n}$ vectors associated with larger subsquares, forms a basis for the projections on the subsquares of side $2^{-(n+1)}$, and the argument proceeds.

We now define $D_{1}$ as the diagonal operator in $H_{1}$ which takes $\varphi_{n}$ into $z_{n} \varphi_{n}$ where $z_{n}$ is the midpoint of the subsquare associated with $\varphi_{n}$.

We now see that $C_{1}=A-D_{1}$ as an operator from $H_{1}$ into $H$ is compact by exploiting two observations:

1. If $\varphi_{n}$ is associated with a subsquare of side $2^{-n}$, then $\left\|C_{1} \varphi_{n}\right\|<2^{-n}$.

2. If $\varphi_{i}$ and $\varphi_{j}$ are associated with distinct subsquares of the same size, then $C_{1} \varphi_{i} \perp C_{1} \varphi_{j}$

These allow us to get a bound of $3 \cdot 2^{-n}$ for $C_{1}$ in the $3 \cdot 2^{n}$ dimensional subspace spanned by all the basis vectors associated with the subsquares of side $2^{-n}$.

There is one trivial exception. In order to start the construction we may need 4 vectors with support the whole square in order to span the projections on the 4 quarters. Therefore we will make the very crude estimate that the bound for $C_{1}$ is $4 \cdot 2^{-n}$ on these subspaces. We now see that $\left\|C_{1}\right\|<4 \sum_{n=0}^{\infty} 2^{-n}$ and so $C_{1}$ is clearly the norm limit of finite-dimensional operators and so compact.

The necessity of using the observation labeled 2 prevents us from proving that $C_{1}$ is Hilbert-Schmidt. Where we can dispense with this observation, as in the case where the spectrum lies in a rectifiable curve (see Theorem 3 or von Neumann [2]) our construction gives us a Hilbert-Schmidt operator.

We now return to "Let us try..." and write out the details for the construction just described.

We let $\Psi^{(1)}=\omega_{1}$. We let $H_{1}=\bigvee_{i, j} P_{i j} \Psi^{(1)}$.

We define $\varphi_{1}$ in $H_{01}(=H)$ by $\varphi_{1}=\Psi^{(1)}$. We next choose $\varphi_{2}, \varphi_{3}, \varphi_{4}$ in $H_{01}$ so that $\left\{\varphi_{i} \mid i=1, \ldots, 4\right\}$ is an orthonormal basis for $\bigvee_{j=1}^{4} P_{i j} \Psi^{(1)}$. (Some of the $\varphi_{i}$ at this and succeeding stages may be 0 . It is simply more confusing than it is enlightening to re-index in such a case.)

We then choose

$$
\begin{array}{r}
\varphi_{5}, \varphi_{6}, \varphi_{7} \in H_{11}, \quad \varphi_{8}, \varphi_{9}, \varphi_{10} \in H_{12}, \\
\varphi_{11}, \varphi_{12}, \varphi_{13} \in H_{13}, \quad \varphi_{14}, \varphi_{15}, \varphi_{16} \in H_{14}
\end{array}
$$

so that $\left\{\varphi_{i} \mid i=1, \ldots, 16\right\}$ is an orthonormal basis for $\bigvee_{j=1}^{16} P_{2 j} \Psi^{(1)}$. This choice of 
the $\varphi_{j}$ was possible because at the preceding stage we had already spanned $P_{1 j} \Psi^{(1)}$. Therefore to get, say, $P_{21} \Psi^{(1)}$ it was sufficient to add a vector in $H_{11}$.

We continue in this way adding at most 3 elements from $H_{n, j}$ so as to include in our span the 4-dimensional subspace generated by $P_{n+1, k_{1}} \Psi^{(1)}, \ldots, P_{n+1, k_{4}} \Psi^{(1)}$ where the $k_{i}$ are determined by the requirement that $P_{n+1, k_{i}} \Psi^{(1)} \in H_{n, j}$. (That is, to achieve the projection corresponding to one square we add a vector from the space corresponding to the next larger square including it.) In this way we get $\bigvee_{i=1}^{4^{n}} \varphi_{i}=\bigvee_{j=1}^{4^{n}} P_{n, j} \Psi^{(1)}$. We continue and arrive at a basis for $\bigvee_{i, j} P_{i, j} \Psi^{(1)}$. We now note that $\Phi_{0}=\left\{\varphi_{j} \mid j \leqq 4\right\}$ is a set of 4 vectors lying in $H_{01}=H$. For $n \geqq 1$ we have $\Phi_{n}=\left\{\varphi_{j} \mid 4^{n}<j \leqq 4^{n+1}\right\}$ is a set of vectors with at most 3 members in each $H_{n, j}$. We now have $H_{1}=\bigvee_{n=0}^{\infty} \Phi_{n}$.

We now define $D_{1}$. Consider the basis element $\varphi_{j}$. Then for one $r$ we have $\varphi_{j} \in \Phi_{r}$. For that $r$ there exists one $k$ such that $\varphi_{j} \in H_{r k}$. (Note that there are many possible $H_{u, v}$ such that $\varphi_{j} \in H_{u, v}$, but only one choice when we require that $u=r$ and determine $r$ by requiring that $\varphi_{j} \in \Phi_{r}$.) Now define $D_{1}\left(\varphi_{j}\right)=z \varphi_{j}$ where $z$ is the midpoint of $S_{r, k}$. We define $D_{1} H_{1}^{\perp}=0$. We observe that $\left\|\left(A-D_{1}\right) \varphi_{j}\right\|<2^{-r}$. But more important, for any unit vector $X_{r}$ in $K_{r}=\bigvee \Phi_{r}$ we see that $\left\|\left(A-D_{1}\right) X_{r}\right\| \leqq 4 \cdot 2^{-r}$ since there are at most 4 members of $\Phi_{r}$ in any $H_{r k}$ and $A H_{r k} \perp A H_{r l}$ for $l \neq k$. Therefore for unit $X$ in $H_{1}$ we have $\left\|\left(A-D_{1}\right) X\right\|<4 \sum_{r=0}^{\infty} 2^{-r}$. Similarly for unit $X$ in $H_{1}$ $\cap\left(K_{0} \vee \cdots \vee K_{n}\right)^{\perp}$ we have $\left\|\left(A-D_{1}\right) X\right\|<4 \sum_{r=n+1}^{\infty} 2^{-r}$. Now since $K_{0} \vee \cdots \vee K_{n}$ is finite dimensional we see that $\left.\left(A-D_{1}\right)\right|_{H_{1}}$ is a compact operator from $H_{1}$ to $H$. Therefore $A P_{1}-D_{1}=C_{1}$ is compact.

We now choose $\Psi^{(2)}$ to be the normalized component of $\omega_{2}$ in $H_{1}^{\perp}$. This implies that $P_{i j} \Psi^{(2)} \perp H_{1}$ since $H_{1}$ is closed under the $P_{i, j}$. We proceed just as before to form an orthonormal basis for $H_{2}=\bigvee_{i, j} P_{i j} \Psi^{(2)}$. (Note that $H_{2} \perp H_{1}$.) However, this time we start with $P_{11} \Psi^{(2)}, P_{12} \Psi^{(2)}, P_{13} \Psi^{(2)}, P_{14} \Psi^{(2)}$, suitably normalized, as our initial basis vectors. Observe that they are already orthogonal to each other and to $H_{1}$. (The purpose of constructing $H_{1}$ closed under the $P_{i j}$ was to allow us to start at this stage with orthogonal vectors of smaller support and yet be sure that $\Psi^{(2)}$ was in the span.) We also note that $\omega_{2} \in H_{1} \vee H_{2}$.

Proceeding as in the $\Psi^{(1)}$ case we get $A P_{2}=D_{2}+C_{2}$, where $D_{2}$ is again a diagonal operator with nonzero eigenvalues only for those vectors in the basis of $\mathrm{H}_{2}$ and $C_{2}$ is again compact. However, this time we get the estimate $\left\|C_{2}\right\|<4 \sum_{n=1}^{\infty} 2^{-n}$.

In general when we get to $\Psi^{(n+1)}$, the component of $\omega_{n+1}$ perpendicular to $\bigvee_{i=1}^{n} H_{i}$, we see that $P_{i j} \Psi^{(n+1)} \perp \bigvee_{i=1}^{n} H_{i}$. In this case we start with normalized $P_{n, 1} \Psi^{(n+1)}, \ldots, P_{n, 4^{n}} \Psi^{(n+1)}$ as our initial basis vectors and get $\left\|C_{n+1}\right\| \leqq 4 \sum_{m=n}^{\infty} 2^{-m}$.

It is clear that $\sum_{n=1}^{\infty}$; strong limit $A P_{n}=A$. Therefore $A=\sum_{n=1 \text {; strong limit }}^{\infty} D_{n}+C_{n}$. It is clear that $D=\sum_{n=1}^{\infty}$; strong limit $D_{n}$ is diagonal. But $\sum_{n=1}^{\infty} C_{n}$ is actually a norm convergent sum of compact operators (since $\sum_{p=1}^{\infty} \sum_{n=p}^{\infty} 2^{-n}<\infty$ ) and hence $\sum_{n=1}^{\infty} C_{n}=C$ is actually compact. Thus $A=D+C$ is our desired decomposition. This completes the proof of Theorem 1 .

We note that the diagonalization which serves in Theorem 1 for $T=\int z d E_{z}$ will 
also serve for $f(T)=\int f(z) d E_{z}$ where $f$ is continuous. However, it is not hard to see that this result does not extend, in general, to measurable functions. In particular, in the case where $T$ is multiplication by $x$ on $L^{2}[0,1]$ we can see by a diagonal construction that no single diagonalization can simultaneously serve for all $L^{\infty}$.

We now sketch the somewhat simpler proof mentioned. We first prove, by a construction similar to that used in Theorem 1, that if $\left\{P_{n}\right\}$ is a commuting family of projections on a separable Hilbert space then there is a decomposition $P_{n}$ $=Q_{n}+K_{n}$ and an orthonormal basis $\left\{\phi_{n}\right\}$ such that

(1) all the $Q_{n}$ are simultaneously diagonalized by $\left\{\phi_{n}\right\}$,

(2) if $P_{n} \perp P_{m}$ then $Q_{n} \perp Q_{m}$,

(3) $K_{n}$ is finite dimensional and $\left\|K_{n}\right\| \leqq 2$.

We now choose the $\left\{P_{n}\right\}$ to be projections on the squares of side $2^{-m}$. We then prove Theorem 1 by approximating $\int z d E_{z}$ by a sequence (in $n$ ) of operators of the form $\sum \lambda_{j(n)} P_{j(n)}$ where the $P_{j(n)}$ are projections on disjoint squares of side $2^{-n}$ and $\lambda_{j(n)}$ is an appropriate scalar. We then use the orthogonality of the $Q_{j(n)}$ for fixed $n$ to see that $\sum \lambda_{j(n)} K_{j(n)}$ sequence really does converge in uniform norm and hence has a compact limit and $\sum \lambda_{j(n)} Q_{j(n)}$ converges (strongly) to a diagonal operator.

However, this proof loses the precise bound on the error which our more exacting construction of the $\left\{\phi_{n}\right\}$ allows. In particular, having a Cauchy sequence of finitedimensional operators is no help in establishing the Hilbert-Schmidt character of the limit.

Corollary 2. Let $A$ be a (not necessarily bounded) normal operator on the separable Hilbert space $H$. Then for $\varepsilon>0$ there exist a diagonal operator $D$ and $a$ compact operator $C$ with norm less than $\varepsilon$ such that $A=D+C$.

Proof. We first note that $\|C\|$ in the case where $\sigma(A)$ lies in the unit square can be made arbitrarily small by starting with small enough squares in the $H_{1}$ step in the proof of Theorem 1. In the general case we pave the whole plane with disjoint unit squares, closed on the left and bottom, open on the right and top. We apply Theorem 1 to the Hilbert space gotten by projection on these squares, requiring the compact operator to have norm bounded by $\varepsilon / 2^{n}$ on the $n$th such square. The fact that each of these spaces may have the top and right of its square in its spectrum is irrelevant; the direct sum of the diagonal operators still yields the appropriate diagonal operator. The direct sum of the compact operators is norm convergent and so compact. For the reader who is squeamish about unbounded operators, we point out that the canonical construction of the spectral form for an unbounded operator is as a direct sum of bounded operators. Of course, while our diagonal operator is defined on a complete set of eigenvectors, it is impossible for it to be defined on all of $H$ in the unbounded case.

THEOREM 3. Let $A$ be a normal operator on $l^{2}$. Let $\sigma(A)$ lie in a curve of finite length. Then we may write $A=D+K$ where $D$ is diagonal and $K$ is Hilbert-Schmidt. 
Moreover for any $\varepsilon>0$ we may select $D$ and $K$ so that the Hilbert-Schmidt norm of $K$ is less than $\varepsilon$.

Proof. Call the finite curve $\Gamma$, and suppose it is of length 1 . Follow the construction used in Theorem 1. However, instead of breaking up the square into successive quarters, break up $\Gamma$ into successive halves, parametrized by length. Then, in assigning projections to pieces of length $1 / 2^{n}$ of $\Gamma$, make the trivial modification of assigning a projection to the first such piece in which it falls in case $\Gamma$ is self intersecting. The spectrum is compact and the pieces of $\Gamma$ lack at most one point to be compact; therefore measurability problems do not arise.

We assign to each of our new basis vectors multiplication by the coordinate of its midpoint and call our new operator $D$.

Now we consider $A-D$ on the $2^{n}$ vectors associated with the partition into $2^{n}$ arcs of length $2^{-n}$. But if $\varphi$ is such a unit vector $\|(A-D)(\varphi)\|^{2} \leqq 2^{-2 n}$. Hence, on this set of vectors we have a Hilbert-Schmidt norm of $2^{-n}$. From here on the estimates proceed just as in Theorem 1, then as in Corollary 2 for the improvement to $\varepsilon$. This completes the proof of Theorem 3 .

COROllaRy 4. Let A be a normal operator on $l^{2}$. Let $\sigma(A)$ lie in a rectifiable curve. Then for $\varepsilon>0$ there exist diagonal $D$ and Hilbert-Schmidt $K$ with Hilbert-Schmidt norm less than $\varepsilon$ such that $A=D+K$.

Proof. We apply Theorem 2 to arcs of length 1 , using bounds of $\varepsilon / 2^{n}$ on the successive Hilbert-Schmidt operators and taking the direct sum of the operators so constructed. Once again we assign spectrum to the first arc in which it lies so as to keep the successive operators on orthogonal subspaces. This completes the proof of Corollary 4.

Corollary 4 clearly implies von Neumann's Theorem 1 of [2]. More interesting is the case where the spectrum lies on a rectifiable curve of infinite length even in a bounded region.

Let us now consider the problem of getting a Hilbert-Schmidt remainder in the general case. The difficulty arises from the fact that we need $2^{2 n}$ squares of side $2^{-n}$ to cover the unit square. Therefore the Hilbert-Schmidt norm of the operator associated with one covering of the square by squares of side $2^{-n}$ may be of the order of $2^{2 n}\left(2^{-n}\right)^{2}=1$. If we consider the operator given by multiplication by $z$ on $L^{2}[I \times I]$ we can easily verify that we will get remainders of this order and hence an infinite Hilbert-Schmidt norm, no matter with what basis vectors $\left\{\omega_{n}\right\}$ we start.

However, we have no proof that our diagonalization is the most efficient possible and so we have to reckon with the possibility that some cleverer diagonalization may have a Hilbert-Schmidt remainder. We do not think that the problem of too much area in the (continuous) spectrum can be surmounted; however, we have no proof. 
Added in proof. W. Sikonia has independently proved Theorem 1 using methods somewhat similar to ours (preprint), and P. R. Halmos has proved Theorem 1 using quite different methods (preprint).

\section{REFERENCES}

1. P. R. Halmos, Ten problems in Hilbert space, Bull. Amer. Math. Soc. 76 (1970), 887-933.

2. J. von Neumann, Charakterisierung des Spektrums eines Integraloperators, Actualités Sci. Indust., no. 229, Hermann, Paris, 1935.

3. H. Weyl, Uber beschränkte quadratischen Formen deren Differenz vollstetig ist, Rend. Circ. Mat. Palermo 27 (1909), 373-392.

UNIVERSITY OF ILLINOIS,

URBANA, ILLINOIS 61801

QUEEN'S UNIVERSITY,

Kingston, Ontario, Canada 